\section{Envolvimento Paterno e Temperamento Infantil: Revisão Sistemática de Literatura}

\author{
Mariana Effting de Sousa Schmitz \\ Meiridiane Domingues de Deus \\ Andressa Pires Gouvêa \\ Sara Bueno da Silva \\ Mauro Luís Vieira
}

\section{RESUMO}

Este estudo tem como objetivo apresentar uma revisão sistemática da literatura sobre envolvimento paterno e temperamento infantil, entre os anos de 2006 e 2017. Realizouse uma busca nas seguintes bases de dados: LILACS, PePSIC, SciELO, Web of Science e PsycINFO (APA), por meio dos descritores father e temperament e seus respectivos termos em português e espanhol. Foram examinados 19 artigos cujo foco de investigação foram a relação entre o envolvimento do pai e o temperamento do(a) filho(a). Os resultados foram divididos em duas categorias: parentalidade, avaliação e influência no temperamento infantil e paternidade e temperamento infantil. Verificou-se que o tema vem sendo pouco estudado no Brasil, em comparação com outros países. A partir dos estudos analisados, pode-se inferir que o pai tem influência direta em todas as fases do desenvolvimento infantil e no temperamento do(a) filho(a), especialmente no que concerne a socialização e regulação do humor. Portanto, aponta-se para a necessidade de ampliação de pesquisas com este foco, a fim de favorecer e estimular a elaboração de políticas públicas e possibilitar novos modos de fomentar o envolvimento paterno na vida dos(as) filhos(as), considerando as variáveis que interferem nesse fenômeno, como o temperamento da criança.

Palavras-chave: Pai. Temperamento. Revisão Sistemática da Literatura.

\section{ABSTRACT}

\section{Paternal Involvement and Child Temperament: Systematic Review Of Literature}

This study aims to present a systematic review of the literature on paternal involvement and child temperament, between the years of 2006 and 2017. A search was carried out in the following databases: LILACS, PePSIC, SciELO, Web of Science and PsycINFO (APA), through the descriptors father and temperament and their respective terms in Portuguese and Spanish. Nineteen articles were examined whose focus of investigation was the relationship between the involvement of the father and the temperament of the child. The results were divided into two categories: parenthood, evaluation and influence on child's temperament and fatherhood and child temperament. It was verified that the subject has been insufficiently researched in Brazil, in comparison with other countries.

\author{
Sobre os Autores \\ M.E.S.S \\ http://orcid.org/0000-0002- \\ 9931-790X \\ Universidade Federal de \\ Santa Catarina, Florianópo- \\ lis, Santa Catarina \\ efftingmariana@gmail.com \\ M.D.D \\ http://orcid.org/0000-0002- \\ 9322-0162 \\ Universidade Federal de \\ Santa Catarina, Florianópo- \\ lis, Santa Catarina \\ meiridomingues@hotmail. \\ com
}

\section{A.P.G}

http://orcid.org/0000-00022509-2584.

Universidade Federal de

Santa Catarina, Florianópo-

lis, Santa Catarina

dessa.gouvea@hotmail.

com

\section{S.B.S}

http://orcid.org/0000-0003-

1580-1516

Universidade Federal de

Santa Catarina, Florianópo-

lis, Santa Catarina

sara.b2294@gmail.com

M.L.V

http://orcid.org/0000-00030541-4133

Universidade Federal de

Santa Catarina, Florianópolis, Santa Catarina

maurolvieira@gmail.com

\section{Direitos Autorais}

Este é um artigo de acesso aberto e pode ser reproduzido livremente, distribuído, transmitido ou modificado, por qualquer pessoa desde queu sado sem fins comerciais. O trabalho é disponibilizado sob a licença Creative Commons CC-BY-NC. 


\section{*ZI' INTERACÃO EM ITS PSICOLOGIA}

From the studies analyzed, it can be inferred that the father has a direct influence on all phases of child development and on the child temperament, especially regarding socialization and mood regulation. Therefore, it is necessary to expand researches with this focus in order to favor and stimulate the elaboration of public policies and to enable new ways to foster paternal involvement in the children's lives, considering the variables that interfere in this phenomenon, such as the temperament of the child.

Keywords: Father; Temperament; Systematic Review of Literature.

Compreender as diferenças nos traços e nas características da personalidade do indivíduo desperta 0 interesse de pesquisadores desde a Antiguidade. 0 conceito de temperamento tem origem em 400 a.C., quando Galeno e Hipócrates propuseram diferentes tipos, considerados os quatros temperamentos primários, a saber: tipo sanguíneo, colérico, melancólico e fleumático (Ito \& Guzzo, 2002). Porém, o estudo deste fenômeno passou a ser mais efetivo no século $\mathrm{XX}$, por meio de pesquisas realizadas por psiquiatras e psicólogos, a partir das décadas de 1950 e 1960 (Guzzo et al., 2004; Ito \& Guzzo, 2002).

0 temperamento apresenta diferentes definições, dimensões e instrumentos para sua avaliação. Entretanto, parece haver um consenso de que o temperamento é considerado como diferenças individuais que aparecem desde a infância (Ito \& Guzzo, 2002; Schmidt, 2012). Os estudos diferem na ênfase que é atribuída aos fatores biológicos e contextuais, assim como na origem do temperamento (Klein \& Linhares, 2007). O fenômeno consiste em um conjunto de características individuais com caráter genético e neurobiológico, influenciado pelas experiências de vida dos indivíduos ao longo do seu desenvolvimento (Prior et al., 2008). Relaciona-se às diferenças na constituição de estilos comportamentais que podem ser perceptíveis em idades precoces, ou seja, no período da infância (Sanson et al., 2004).

No que tange o temperamento infantil, pesquisas vêm sendo realizadas para compreender a base do desenvolvimento da personalidade, a repercussão no ciclo de vida e a influência das figuras parentais na sua constituição (Cassiano, 2013; Klein, 2009; Schmidt, 2012; Schmidt et al., 2019). As figuras parentais desempenham papéis diferentes na criação e no desenvolvimento da criança, conforme o contexto cultural. Os papéis maternos e paternos são multidimensionais, complexos, e a modificação destes papéis alteram as responsabilidades, as tarefas com o(a) filho(a) e, consequentemente, as tarefas domésticas. Tradicionalmente, as mães são identificadas como cuidadoras primárias do(a) filho(a), já o pai, como provedor econômico da família, desempenhando um papel secundário nos cuidados com os(as) filhos(as) e nas tarefas domésticas (Bossardi \& Vieira, 2015).

No decorrer dasúltimas décadas, mudanças relevantes ocorreram nas dinâmicas familiares e pesquisas relacionadas à Psicologia do Desenvolvimento, acerca da paternidade e do papel paterno no desenvolvimento infantil, tornaram-se recorrentes (Goetz \& Vieira, 2010). A importância do pai para o desenvolvimento infantil passou a ser evidenciada a partir da década de 1970, com Michael Lamb e a publicação do livro "O papel do pai no desenvolvimento infantil", em 1976 (Goetz \& Vieira, 2010). Diante disto, as pesquisas relacionadas à importância do pai no desenvolvimento infantil utilizam do termo envolvimento/engajamento paterno para investigar tais fenômenos (Backes, 2015; Bolze, 2011).

O envolvimento paterno é um termo proposto por Lamb et al. (1985). É caracterizado e definido através de três dimensões: a) interação: tempo que se passa em interação efetiva com a criança, como alimentar e brincar com a mesma; b) acessibilidade: presença e disponibilidade do pai com a criança, como atividades que envolvam graus menos intensos de interação, e; c) responsabilidade: garantir cuidados e recursos para a criança, assumindo responsabilidade pelo bem-estar e cuidados (Lamb, 1992).

O pai oferece um cuidado diferente do cuidado materno. A ligação entre mãe e criança é baseada no conforto, proteção e segurança, e os mecanismos envolvidos na ligação entre o pai e a criança são outros (Bolze, 2011; Paquette, 2004a). Por sua vez, os pais tendem a contribuir para a socialização da criança e a função de abertura ao mundo (Bolze, 2011; Paquette, 2004a; Paquete \& Dumont, 2013). Os pais têm estado mais participativos nas atividades com o(a) filho(a), bem como na transmissão de confiança e segurança, tornando-se mais próximos da sua família e dos(as) filhos(as) (Silva et al., 2014). Assim, a presença e participação contínua do pai é essencial para o bem-estar físico e psicológico, e também, na constituição do temperamento da criança, nas diferentes fases de seu desenvolvimento.

No que se refere ao estado da arte em pesquisas relacionadas à temática do temperamento infantil, cita-se 

Andressa Pires Gouvêa, Sara Bueno da Silva, Mauro Luís Vieira

a revisão realizada por Guzzo et al. (2004) que investigaram publicações contemporâneas sobre o temperamento e a sua relação com a Psicologia do Desenvolvimento, e a revisão sistemática de Linhares et al. (2013) que objetivou investigar as publicações referentes ao temperamento de crianças no período do nascimento até a idade escolar, de acordo com a abordagem de Rothbart. A revisão proposta por Consentino-Rocha e Linhares (2013) analisou estudos que se referiam ao efeito do temperamento e gênero no desenvolvimento, do nascimento até a idade escolar. Gracioli e Linhares (2014) revisaram de forma sistemática estudos empíricos sobre temperamento e suas relações com problemas emocionais e de comportamento em crianças na fase pré-escolar. Schmidt et al. (2019) analisaram sistematicamente a produção científica sobre as relações entre envolvimento parental e temperamento de crianças, nos últimos 10 anos.

Entretanto, a avaliação do temperamento das crianças pré-escolares ainda é um tema pouco encontrado nas publicações científicas, o que aponta para a importância de compreender como esse fenômeno se desdobra, incluindo a participação e a relação do pai em sua constituição (Schmidt, 2012; Seabra-Santos \& Almeida, 2014). Neste sentido, a relevância científica deste artigo reside na ampliação de literatura referente a esta temática, por possibilitar um avanço nas pesquisas do cenário brasileiro referente ao temperamento de crianças pré-escolares.

Ademais, compreender a relação existente entre o envolvimento paterno e o temperamento do(a) filho(a) é relevante socialmente por possibilitar a elaboração de políticas públicas e projetos de intervenção a fim de fomentar o envolvimento do pai na vida dos(as) filhos(as), considerando as variáveis psicossociais que interferem nesse fenômeno. Pode-se, ainda, fomentar a promoção de intervenções psicoterápicas com as diferentes configurações familiares. Deste modo, o objetivo desse estudo é apresentar uma revisão sistemática da literatura sobre envolvimento paterno e o temperamento infantil, entre os anos de 2006 e 2017.

\section{MÉTODO}

No mês de agosto de 2017, procedeu-se às buscas de artigos indexados nas bases de dados eletrônicas LILACS, PePSIC, SciELO, Web of Science e PsycINFO (APA). A escolha dos descritores utilizados ocorreu inicialmente pela Terminologia em Psicologia da BVS, mas observou-se que o termo "temperamento" é utilizado como sinônimo de "personalidade". Assim, em função das diferenças entre esses conceitos, optou-se por realizar as buscas através dos Descritores em Ciências da Saúde (DeCS), tal como foi realizado no estudo de Schmidt et al. (2011). Os descritores indexados utilizados para a pesquisa foram "pai" e "temperamento", considerando suas versões em língua inglesa e espanhola. A pesquisa ocorreu pela combinação dos termos, sempre no mesmo idioma, recorrendo-se ao operador lógico AND.

Os critérios de inclusão utilizados na busca consistiram em: A) acesso aos resumos e textos integrais nos idiomas português, inglês ou espanhol; B) ter sido publicado no período de 2006 a 2017; C) ser artigo indexado em periódico científico; D) ser artigo de abordagem metodológica quantitativa; E) investigar os temas envolvimento paterno e temperamento infantil; F) analisar de maneira independente dados relativos à figura paterna, quando ambas as figuras parentais forem investigadas. A delimitação desse período de publicação se justifica pelas transformações no envolvimento paterno nas famílias contemporâneas, em que a participação paterna nos cuidados à criança é crescente (Vieira et al., 2014) Os estudos envolvendo a avaliação do temperamento infantil, em sua maioria, utilizam escalas e questionários como método de análise quantitativo para compreender tal fenômeno. Por este motivo, delimitou-se como critério de inclusão os estudos com abordagem quantitativa. Já os critérios para exclusão: a) textos de livros, jornais e revistas não científicas, teses e dissertações, trabalhos apresentados em congressos (como resumos, trabalhos e/ou conferências); b) artigos não disponibilizados na íntegra e idiomas elencados, c) artigos de revisão ou de abordagem qualitativa; d) artigos que não se relacionem aos temas envolvimento paterno e temperamento infantil; e) artigos com acesso restrito (pagos).

A partir das buscas nas plataformas de dados, foram encontrados 441 artigos. Realizou-se a organização e tabulação dos artigos por meio do programa Microsoft Excel. Primeiramente, foram excluídos 79 artigos duplicados, 25 artigos com acesso restrito (pagos) e sem acesso ao resumo e ao texto na íntegra. Procedeuse à leitura dos resumos dos demais 337 artigos. Foram excluídos, após leitura dos resumos, 318 estudos que não contemplavam os critérios de inclusão elencados. Por fim, foram selecionados 19 artigos que investigaram os temas que a pesquisa objetivou estudar. 

Andressa Pires Gouvêa, Sara Bueno da Silva, Mauro Luís Vieira

Todo o processo foi realizado sob análise de dois juízes independentes, por meio da concordância entre os juízes. Utilizou-se a mesma escala de avaliação, em que cada juiz fornecia uma classificação sobre o artigo analisado, o que permitiu reduzir os vieses e buscar o consenso nos estudos incluídos para análise integral (Matos, 2014). A Figura 1 descreve o percurso metodológico de seleção dos artigos conforme as recomendações do protocolo PRISMA.

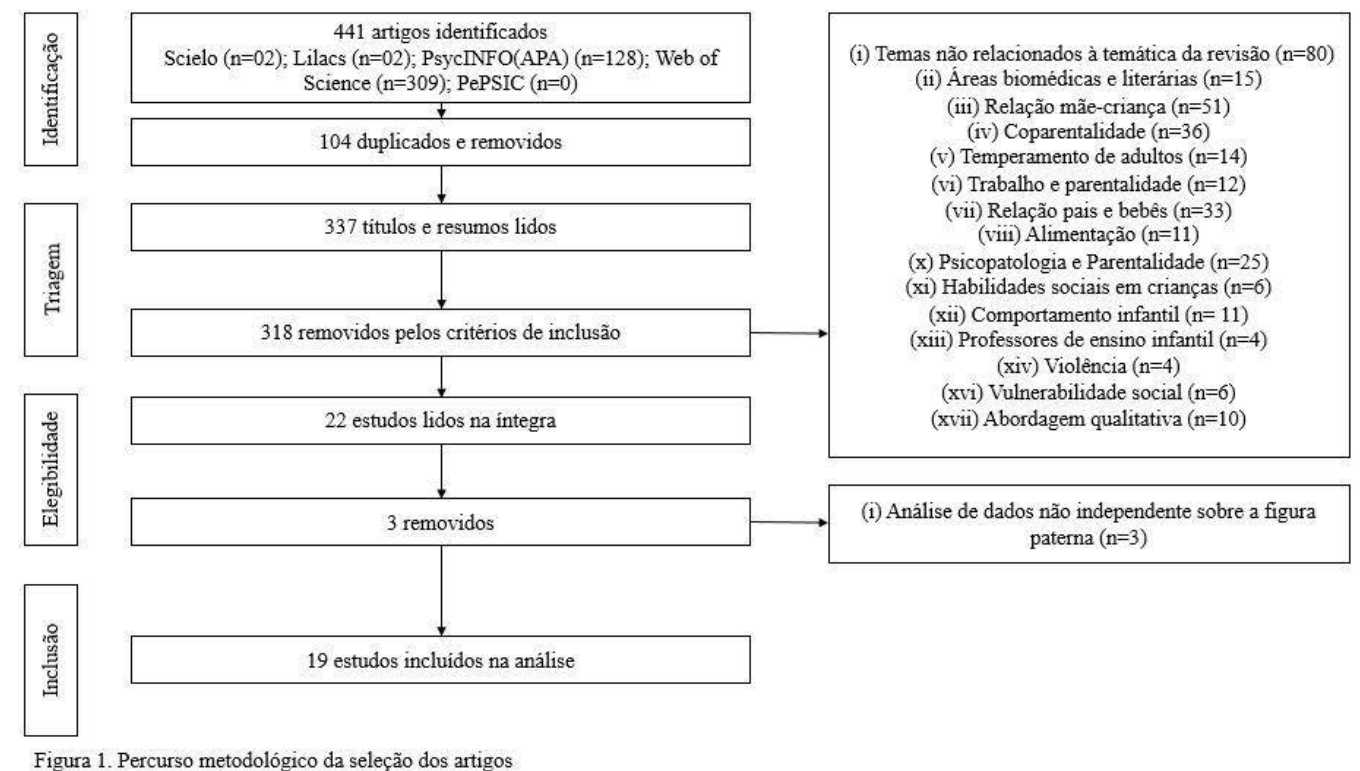

A elaboração das categorias ocorreu por meio da leitura integral dos 19 artigos. Após a leitura integral dos artigos, realizou-se a classificação e diferenciação dos conteúdos abordados em cada estudo, em seguida, avaliou-se pertinência dos conteúdos ao objetivo dessa revisão e, por fim, foi realizado o agrupamento dos temas afins (Oliveira, 2008). Os artigos estruturam-se na breve caracterização dos estudos encontrados, seguidos pelas seguintes categorias: (1) parentalidade, avaliação e influência no temperamento infantil e (2) paternidade e temperamento infantil.

\section{RESULTADOS}

\section{Caracterização dos estudos}

Após a leitura integral dos artigos selecionados, constatou-se que os participantes dos estudos selecionados foram, em sua maioria, a tríade pai/mãe/ filho (15 estudos), a percepção de pai/mãe/professores sobre o temperamento infantil foi investigada em dois estudos. Por sua vez, a díade pai-filho foi averiguada em dois materiais analisados. Cabe ressaltar que a inclusão dos trabalhos analisados que investigaram a família de maneira ampla (pai/mãe/filho) deu-se por discutirem e analisarem a influência das figuras parentais no temperamento da criança de maneira independente, permitindo, assim, a inserção destes estudos na presente análise. Tais resultados demonstram que há escassez de estudos que abordam especificamente a díade pai-filho.

No que tange o tipo de estudo, foram encontrados 10 estudos com corte transversal e 9 longitudinais. Os estudos longitudinais foram desenvolvidos com as figuras parentais em que a criança era alvo da avaliação do temperamento (Kim \& Kochanska, 2012). Com relação 
à idade das crianças, os sujeitos alvos das avaliações sobre o temperamento, percebeu-se que 15 pesquisas referiam ao temperamento de crianças de zero a 36 meses. Crianças em idade pré-escolar e escolar foram foco de três estudos, e pré-adolescentes foram alvo de um estudo.

Os países em que os estudos foram efetuados são: Portugal $(n=1)$, Japão $(n=1)$, Canadá $(n=1)$, Itália $(n=1)$, Finlândia $(n=1)$, Holanda $(n=1)$, Reino Unido $(n=3)$ e Estados Unidos $(n=10)$. Assim, a maior parte dos estudos foram executados na América do Norte e Europa. Destacase que não foram encontrados estudos brasileiros sobre a temática nesta revisão, dado de grande relevância considerando a importância dessa temática para o desenvolvimento infantil.

o método predominante foi o levantamento de dados, seguido pela combinação entre levantamento de dados e observação. Os instrumentos de coleta de dados mais utilizados foram questionários, escalas, roteiros de observação em laboratório e na residência e a combinação entre a aplicação de questionários e/ ou escalas e observação. As medidas utilizadas para a investigação do temperamento infantil, em seis pesquisas averiguadas, foram questionários desenvolvidos com base na abordagem teórico-conceitual de Rothbart, CBQ - Child Behavior Questionnaire, para crianças de 3 a 7 anos, e o IBQ - Infant Behavior Questionnaire, para crianças de 3 a 12 meses de idade. Nas demais pesquisas, recorreu-se aos roteiros de observação na avaliação de temperamento e demais questionários que investigam o temperamento a partir de outras perspectivas, como o The Italian Questionnaires on Temperament, empregado na pesquisa de Cerniglia et al. (2014).

Os principais resultados obtidos na leitura dos artigos foram agrupados nas seguintes categorias: parentalidade, avaliação e influência no temperamento infantil e paternidade e temperamento infantil.

\section{Parentalidade, avaliação e influência no temperamento infantil}

Constatou-se que não há diferenças significativas entre as figuras parentais na avaliação do temperamento do(a) filho(a). Isto é, a percepção de pais e mães sobre o temperamento da criança não é desigual (Casalin et al., 2012; Kitamura et al., 2015; Seabra-Santos \& Almeida, 2014). Ao que concerne à diferença de gênero na avaliação do temperamento, Casalin et al. (2012) apontam não haver diferenças significativas entre meninas e meninos, nos três fatores do temperamento.

A avaliação paterna do temperamento da criança baseia-se, principalmente, no período que pai e filho interagem nos jogos e brincadeiras (Kim \& Kochanska, 2012; Komsi et al., 2008). Por outro lado, as mães informam sobre o temperamento do(a) filho(a) com base na interação dos cuidados primários com o(a) filho(a) (Cerniglia et al., 2014). Assim, a verificação de não divergência pode ser compreendida pelo tempo que tanto a mãe quanto o pai passam em interação direta com a criança (Seabra-Santos \& Almeida, 2014).

No que tange ao tempo passado em interação com a criança, tanto pais quanto mães parecem passar mais tempo com crianças com temperamento desafiador do que com crianças com temperamento mais fácil, em dias úteis, em que os pais estão envolvidos com suas atividades laborais (Brown et al., 2011). Porém, os pais passaram menos tempo em interação com crianças de temperamento desafiador, em dias não úteis, ou seja, nos fins de semana.

Em relação às avaliações do temperamento infantil realizadas por professores, verificou-se que há diferenças significativas da análise de pai e mãe. Esse dado pode ser apreendido pelo pouco tempo de convívio diário com as crianças, uma vez que o parâmetro de avaliação foi a interação e o tempo passado em sala de aula com a criança (Seabra-Santos \& Almeida, 2014). Na pesquisa realizada por Karreman et al. (2010), o relato dos professores referente ao temperamento apresentado pelas crianças durante o período passado nas creches foi estudado como complemento da análise.

Aponta-se, ainda, que o temperamento da criança parece ser influenciado pelo estilo de interação com pai e mãe (e vice-versa), de maneira que, quanto mais próximo e envolvido com o(a) filho(a), mais segura e assertiva será sua avaliação sobre o temperamento do(a) filho(a), assim como a percepção sobre as necessidades da criança (Seabra-Santos \& Almeida, 2014). Nesse aspecto, o estudo de Karreman et al. (2010) constata não haver diferenças significativa na influência de ambas figuras parentais no comportamento e no temperamento da criança. Entretanto, verificou-se que o pai desempenha um papel importante em fatores específicos do temperamento infantil, tais como socialização e regulação do humor (Karreman et al., 2010), e a relação pai-filho é considerada um moderador do temperamento (Aviram et al., 2015; Kochanska \& Kim, 2014).

A avaliação e a influência das figuras parentais 

Andressa Pires Gouvêa, Sara Bueno da Silva, Mauro Luís Vieira

no temperamento da criança ocorrem de maneira semelhante, conforme os estudos analisados. Porém, o pai tem influência mais significativa em aspectos específicos relacionados ao temperamento da criança, como a socialização e regulação do humor. Esses aspectos serão apresentados na categoria "Paternidade e temperamento infantil”.

\section{Paternidade e temperamento infantil}

Os estudos sobre os efeitos do temperamento infantil na interação pai-filho, e vice-versa, demonstram que há associação entre o envolvimento do pai com as dificuldades infantis (Komsi et al., 2008; Planalp \& Braungart-Rieker, 2013). Assinalam que o envolvimento paterno é influenciado pelos comportamentos e temperamento dos(as) filhos(as) (Brown et al., 2011; Cerniglia et al., 2014), pela relação conjugal, contexto sociocultural e ecológico (Mehall et al., 2009), traços genéticos (Flouri \& Malmberg, 2010) e se modifica conforme o nível de educação (Komsi et al., 2008). Além desses aspectos, o tempo dispendido em interação com a criança e a jornada de trabalho do pai são considerados fatores moderadores do temperamento infantil (Brown et al., 2011; Kochanska \& Kim, 2014).

Os pais são mais propensos a envolver-se nos cuidados com crianças de mais idade (Mehall et al., 2009; Paquette \& Dumont, 2013) e têm influência mais significativa no temperamento do filho homem, no que diz respeito ao controle de humor (Hanington et al., 2010). A diferença de gênero foi verificada na pesquisa de Hanington et al. (2010) que analisava a influência da depressão paterna no temperamento da criança, concluindo que as crianças de sexo masculino têm maior risco de apresentar dificuldades comportamentais e emocionais do que as filhas. Os autores apontam que essa diferença pode ser explicada pela forma que os pais interagem com o filho homem (e vice-versa), e que essa psicopatologia pode dificultar a tarefa do pai em regular as emoções dos(as) filhos(as).

O tempo passado em interação com a criança e a qualidade desta interação aparecem como fatores importantes na modulação do temperamento infantil. Pais que passam pouco tempo em contato com o(a) filho(a) e com envolvimento pouco sensível têm crianças mais propensas à angústia, dificuldades nas interações sociais e gerenciamento de emoções (Brown et al., 2009). As crianças, ainda, expõem maior tendência por apresentar temperamento difícil, desafiador ou irregular.
Os traços genéticos paternos são fatores moderadores do temperamento infantil. Filhos de pais com temperamento ou comportamentos difíceis são mais propensos a alto risco de comportamento e temperamento difíceis (Flouri\&Malmberg, 2010). Crianças com temperamento $v$ fácil diminuem as chances de os pais distanciarem-se da unidade familiar, assim como aumentam as chances de os pais envolverem-se com os(as) filhos(as). Flouri e Malmberg (2010) informam que as características biológicas, sociodemográficas, fatores contextuais, ambientais e o comportamento do pai influenciam diretamente em aspectos comportamentais e relacionados ao temperamento das crianças.

A parentalidade positiva paterna interagiu com a flexibilidade infantil, no que tange problemas comportamentais internalizantes e externalizantes, componentes do temperamento (Rabinowitz et al., 2016). Verificou-se que a flexibilidade dos pré-adolescentes se modificou conforme a flexibilidade do papel paterno e o tempo em interação com o(a) filho(a), associando a paternidade positiva à flexibilidade temperamental dos(as) filhos(as). Por outro lado, crianças com menor índice de flexibilidade paterna obtiveram um índice maior de sintomas de comportamentos internalizantes e externalizantes (Rabinowitz et al., 2016).

Por outrolado, níveis mais elevados de envolvimento do pai têm sido associados a resultados de desenvolvimento mais positivos em vários domínios, como o melhor gerenciamento das emoções (Bayly \& Garstein, 2013; Brown et al., 2009), no controle e na intensidade do humor (Hanington et al., 2010), na socialização (Brown et al., 2009) e influência na emocionalidade (como raiva, depressão e impulsividade) (Kitamura et al., 2015). Assim, os cuidados, a interação e o tempo dispendido dos pais afetam as características do temperamento (e vice-versa) (Potapova et al., 2014).

\section{DISCUSSÃO}

Os artigos selecionados para essa revisão, em sua maioria, possuem como participantes das pesquisas a tríade pai/mãe/filho. Em contrapartida, há uma escassez de estudos que abordam a díade pai-filho. A realização de pesquisas como múltiplos informantes contribui para a redução de vieses relativos às percepções sobre o temperamento das crianças (Linhares et al., 2013).

A constatação da existência de um baixo número de estudos que investigam a relação pai-filho vai ao encontro dos achados do estudo de revisão realizado 
por Linhares et al. (2013) que teve como objetivo revisar sistematicamente a literatura publicada entre os anos de 2008 a 2011 que tratava do temperamento de crianças no período do nascimento até a idade escolar, considerando a abordagem de Rothbart. Resultou em 25 estudos, sendo que desse total, $92 \%$ possuíam as mães das crianças como principais informantes das pesquisas. As autoras argumentam que a escolha da figura materna como respondente dos estudos é justificada pela proximidade que as mães têm com os(as) filhos(as) e, com isso, podem descrever as características e comportamentos da criança com maior precisão em diferentes contextos. Outros estudos, como os de Alvarenga e Piccinini (2007) e Malhado e Alvarenga (2012), também possuem as mães como informantes nos estudos que investigam o temperamento em bebês.

A idade das crianças-alvo dos estudos encontrados é predominantemente de zero a 36 meses, seguido da idade pré-escolar e escolar. Esses dados vão ao encontro dos achados de Guzzo et al. (2004), Klein (2009), Linhares et al. (2013), Rothbart e Hwang (2002) e Schmidt et al. (2011), ao constatarem que há um maior interesse de pesquisas relacionadas ao temperamento infantil com foco na infância e, em sua maioria, as figuras parentais são os principais informantes. Ressalta-se, ainda, que a investigação do envolvimento paterno é recorrente a partir dos três anos de idade, pois a criança demonstra maior abertura nas relações, além da relação com a mãe. Assim, a relação e a interação do pai com a criança tendem a ser mais ativa quando a criança é mais velha (Lamb et al., 1985).

Em relação aos métodos, os estudos selecionados caracterizam-se pela abordagem metodológica quantitativa e a estratégia de medida do temperamento infantil foi, predominantemente, levantamento de dados, seguido por levantamento de dados e observação. 0 uso de levantamento de dados, ou levantamento de dados juntamente com a observação, também foi algo observado na revisão sistemática realizada por Schmidt et al. (2011) que teve como objetivo analisar a produção científica que abordavam associações entre temperamento dos(as) filhos(as) e relacionamento conjugal dos pais. A observação é uma maneira de examinar fatos e fenômenos que se deseja estudar, assim, pode-se considerar que a combinação entre a aplicação de questionários e a observação direta do temperamento consiste na maneira mais indicada de investigar esse fenômeno.

Quanto aos instrumentos de coleta de dados utilizados, os estudos selecionados na revisão aplicaram questionários/escalas, roteiros de observação em laboratório e na residência e a combinação entre a aplicação de questionários/escalas e observação. Esses resultados estão em conformidade com os achados de Rothbart e Hwang (2002), em que afirmaram que as formas mais usuais de verificação do temperamento infantil caracterizaram-se pela aplicação de questionários e realização de observações em laboratório ou na residência familiar.

0 modelo proposto por Rothbart permite avaliar o temperamento numa perspectiva desenvolvimental, ao possibilitar a mensuração deste construto nas diferentes fases do ciclo de vida do indivíduo (Klein \& Linhares, 2010). Para Rothbart (2004), o temperamento frequentemente se modifica ao longo do curso do desenvolvimento. Assim como na revisão sistemática realizada por Linhares et al., (2013), o número de estudos longitudinais encontrados foi superior aos estudos transversais e aos realizados na América do Norte e Europa, continentes em que as pesquisas relacionadas ao desenvolvimento infantil são recorrentes. Deste modo, a ideia de investigar o temperamento de maneira longitudinal considera que o temperamento pode moldar e ser moldado, por meio da adaptação individual e das diferentes trajetórias de desenvolvimento, isto é, o contexto em que o indivíduo se insere (Rothbart, 2004).

Outra questão relevante no estudo do temperamento relaciona-se ao gênero da criança. O temperamento pode se expressar de modo diferente em relação ao gênero da criança, mais precisamente, nos fatores componentes do temperamento, tal como verificado no estudo de Cosentino-Rocha e Linhares (2013), que tinha como objetivo analisar os estudos sobre o efeito do temperamento e gênero ao longo de uma etapa do desenvolvimento, no caso do nascimento até a idade escolar. A pesquisa realizada por Else-Quest et al. (2006) demonstrou que a diferença de gênero em relação ao temperamento infantil, sobretudo com relação à dimensão de Controle de Esforço, é maior em meninas, enquanto que Atividade e Prazer de Alta Intensidade, relacionados ao fator Extroversão, apresentou-se com maior escore em meninos.

Ainda, a parentalidade exerce influência significativa no temperamento infantil. Alguns estudos sobre temperamento infantil, como exemplo, o estudo realizado por Cook et al., (2009), que examinou as relações entre temperamento da criança, especificamente, afeto negativo e coparentalidade em 111 famílias com crianças de quatro anos, apresentou correlação significativas entre as respostas de pais e mães. Já a pesquisa de Burney e Leerkes (2010), que buscou investigar a percepção de pais e mães sobre temperamento infantil e coparentalidade, 

Andressa Pires Gouvêa, Sara Bueno da Silva, Mauro Luís Vieira

constatou diferenças na avaliação das figuras parentais nas dimensões relativas ao estresse diante de limites e capacidade de se acalmar. De acordo com os autores, as mães disponibilizam de maior tempo junto aos(as) filhos(as) que os pais, e isso justificaria as diferenças nas percepções nessas dimensões.

Os estudos realizados por Lee et al. (2013), Miller et al. (2013) e Nelson (2015) investigaram o temperamento de crianças com relação à parentalidade. Lee et al. (2013) realizaram uma pesquisa com objetivo de examinar as relações bidirecionais entre o temperamento da criança e estilos parentais, em uma amostra de escolares chineses. Foi possível constatar relações bidirecionais entre pais autoritários e crianças com temperamento que evidenciam baixo escores de controle de esforço (raiva e frustração). Em contrapartida, a parentalidade prediz o temperamento da criança, e vice-versa, são mutualmente influenciados.

No estudo de Nelson (2015), concluiu-se que os conflitos familiares afetam o temperamento das crianças, provocando maior reatividade negativa, problemas de comportamentos externalizantes e internalizantes. No que tange o temperamento das crianças, a parentalidade com baixa carga autoritária prevê crianças mais propensas à raiva. Já a parentalidade com alta carga autoritária prediz crianças com menos propensão à raiva (Miller et al., 2013).

0 envolvimento paterno exerce repercussões indiretas no desenvolvimento infantil: ao favorecer o bem-estar da mãe, bom relacionamento conjugal, compartilhar das tarefas domésticas e sustento econômico familiar, o pai contribui para um clima harmônico e positivo no desenvolvimento da criança (Bueno et al., 2015). Assim, o desenvolvimento social e psicológico das crianças é influenciado pelos ambientes em que elas estão inseridas e pela relação que estabelecem suas figuras parentais. 0 envolvimento paterno pode ser mediado pela presença e interferência materna, assim, quanto mais estável for a relação conjugal, maior será o envolvimento do pai com o(a) filho(a) (Bolze, 2011; Bossardi et al., 2016).

Alguns estudos evidenciam que o próprio pai tem entendimento da importância que lhe é atribuída na família, mesmo antes do nascimento de seu filho (Caires \& Vargens, 2012; Nogueira \& Ferreira, 2012; Perdomini \& Bonilha, 2011). Os pais compreendem que a relação que é estabelecida entre pai-bebê inicia-se precocemente, podendo ser um preditor importante para o desenvolvimento do seu filho, tendo intercorrências na vida adulta (Martins et al., 2014).
Cabe ainda pontuar que as características da personalidade da criança exercem influência na maneira como o pai interage e se envolve. Aspectos relacionados ao temperamento da criança são associados aos conflitos conjugais, bem como ao modo como os pais se engajam com a criança (Cassiano, 2013; Schmidt, 2012). O envolvimento paterno é, neste sentido, uma variável que sofre influência direta e indireta do contexto em que a família está inserida, das características do relacionamento conjugal e das particularidades da própria criança, como o temperamento.

Neste contexto, a relação entre a paternidade e temperamento infantil é algo de grande relevância, haja vista que é importante conhecer as implicações e influências paternas no temperamento infantil. A figura paterna tem funções específicas na socialização e controle da agressividade na infância (Cia \& Barham, 2009; Paquette, 2004a; 2004b; Silva \& Piccinini, 2007). Pais e mães possuem funções diferentes e, em algumas questões, o comportamento do pai sobre os(as) filhos(as) têm maior influência quando comparado com o das mães, como no relacionamento conjugal (Bolze, 2011; Bossardi, 2011), no suporte financeiro familiar (Turcotte e Gaudet, 2009) e no temperamento infantil, tal como aponta o estudo de Mehall et al. (2009).

0 estudo realizado por Rabinowitz et al. (2016) ressalta que a parentalidade positiva paterna contribui para a flexibilidade infantil relacionada aos problemas comportamentais internalizantes e externalizantes, componentes do temperamento. Em relação a essas questões, o envolvimento do pai na vida das crianças contribui de modo significativo para a assertividade, controle da raiva, as relações com pares, desempenho escolar e ajustamento social (Lamb, 1992; Paquette, 2004a, 2004b). Além disso, o envolvimento do pai no cuidado com os(as) filhos(as) tem repercussões positivas no desenvolvimento das crianças (Dubeau et al., 2009; Paquette, 2004b).

As influências dos aspectos genéticos da pessoa devem ser consideradas e podem influenciar nas características individuais, assim como no temperamento. Do mesmo modo, o envolvimento do pai na vida do(a) filho(a) é influenciado por diferentes variáveis, relacionadas a família, aspectos socioculturais, contextuais e com as características da própria criança. Portanto, esses fenômenos são mutualmente influenciados entre si e pelas diferentes variáveis envolvidas no ciclo vital da família. 


\section{4*INTERACÃO EM ET PSICOLOGIA}

\section{CONSIDERAÇÕES FINAIS}

A partir desta revisão sistemática de literatura sobre envolvimento paterno e temperamento infantil, considerando artigos científicos publicados entre os anos de 2006 a 2017, verificou-se que esse tema vem sendo pouco estudado no Brasil, em comparação a pesquisadores de diferentes países. Tal evidência é considerada uma limitação na elaboração deste estudo. Os principais achados desta produção referemse à constatação de que há diferentes formas de se avaliar esse tema, assim como as pessoas mais aptas para essa avaliação, como as figuras parentais e professores. Entretanto, pais e mães que convivem constantemente com os(as) filhos(as) são as pessoas que possuem maiores condições de avaliar e influenciar o temperamento da criança.

Os estudos analisados permitem inferir que o pai tem influência direta no desenvolvimento infantil e no temperamento do(a) filho(a), especialmente no que concerne a socialização e regulação do humor. Com relação ao gênero da criança, percebeu-se que meninos apresentaram com maior frequência comportamentos relacionados a prazer de alta intensidade, enquanto meninas comportamentos de controle com esforço.

Salienta-se a importância de estudos que contemplem a figura paterna como principal informante das pesquisas, a fim de compreender sua perspectiva, bem como conhecer as influências e especificidades que permeiam a relação pai-filho e a sua influência no temperamento da criança. Assim, sugere-se a realização de estudos longitudinais que relacionem envolvimento do pai e temperamento da criança e a investigação da influência dos aspectos intergeracionais com foco nesta temática.

Por fim, destaca-se que a ampliação de pesquisas com este foco pode favorecer e estimular a elaboração de políticas públicas a fim de possibilitar novas maneiras de fomentar o envolvimento paterno na vida dos(as) filhos(as), levando em consideração todas as variáveis que interferem nesse fenômeno - como o temperamento da criança. Ainda neste escopo, pode-se pensar na promoção de intervenções psicoterápicas com as diferentes configurações familiares.

\section{DECLARAÇÃO DA CONTRIBUIÇÃO DOS AUTORES}

Certificamos que todos os autores participaram suficientemente do trabalho para tornar pública sua responsabilidade pelo conteúdo. A contribuição de cada autor pode ser atribuída como se segue:

M.E.S.S e M.D.D. contribuíram para a conceitualização, redação, investigação, versão inicial e final do artigo;

A.P.G., S.B.S e M.D.D. foram responsáveis pela investigação e análise de juízes da revisão de literatura realizada.

M.L.V. é responsável pela revisão e edição final.

\section{DECLARAÇÃO DE CONFLITOS DE INTERESSE}

Os autores declaram que não há conflitos de interesse no manuscrito submetido.

\section{REFERÊNCIAS}

Alvarenga, P., \& Piccinini, C. A. (2007). O impacto do temperamento infantil, da responsividade e das práticas educativas maternas nos problemas de externalização e na competência social da criança. Psicologia: Reflexão e Crítica, 20(2), 314-323. http:// dx.doi.org/10.1590/S0102-79722007000200018

Aviram, I., Atzaba-Poria, N., Pike, A., Meiri, G., \& Yerushalmi, B. (2015). Mealtime dynamics in child feeding disorder: the role of child temperament, parental sense of competence, and paternal involvement. Journal of Pediatric Psychology, 40(1), 45-54. https:// doi.org/10.1093/jpepsy/jsu095

Backes. M.S.(2015). A relação entre o envolvimento paterno e a abertura ao mundo em pais de crianças entre quatro a seis anos (Dissertação de Mestrado, Universidade Federal de Santa Catarina). Repositório Institucional da UFSC. https://repositorio.ufsc.br/ xmlui/bitstream/handle/123456789/133087/333649. pdf?sequence $=1$ \&isAllowed $=y$

Bayly, B., \& Garstein, M. (2013). Mother's and father's reports on their child's temperament: does gender matter? Infant Behavior and Development, 36(1), 171175. http://dx.doi.org/10.1016/j.infbeh.2012.10.008 
Bolze, S. D. A. (2011). A relação entre engajamento paterno e qualidade de relacionamento conjugal de pais com crianças de 4 a 6 anos. (Dissertação de Mestrado, Universidade Federal de Santa Catarina). Repositório Institucional da UFSC https://repositorio.ufsc.br/ xmlui/bitstream/handle/123456789/96041/298985. pdf? sequence $=1$ \&isAllowed $=y$

Bossardi, C. N. (2011). Relação do engajamento parental e relacionamento conjugal no investimento com os filhos. (Dissertação de Mestrado, Universidade Federal de Santa Catarina). Repositório Institucional da UFSC https://repositorio.ufsc.br/xmlui/ handle/123456789/95383

Bossardi, C.N., Gomes, L. B., Bolze, S. D. A., Crepaldi, M. A., \& Vieira, M. L. (2016). Desafios de ser pai em uma sociedade em transformação. In L. V. C. Moreira, E. P. Rabinovich \& P. C. S. V. Zucoloto (Eds). Paternidade na sociedade contemporânea: o envolvimento paterno e as mudanças da família (pp. 81-100). Juruá.

Bossardi, C. N., \& Vieira, M. L. (2015). Ser mãe e ser pai: integração de fatores biológicos e culturais. In E. R. Goetz e M. L. Vieira (Eds). Novo Pai: percursos, desafios e possibilidades (pp.15-30). Juruá.

Bronfenbrenner, U. (1996). A ecologia do desenvolvimento humano. Artes Médicas.

Brown, G. L., Mangelsdorf, S. C., Neff, C., SchoppeSullivan, S. J. e, Frosch, C. A. (2009). Young children's self-concepts: Associations with child temperament, mother's and father's parenting, and triadic family interaction. Merrill-Palmer Quarterly, 55(2), 184-216. http://dx.doi.org/10.1353/mpq.0.0019

Brown, G. L., McBride, B. A., Bost, K. K., e Shin, N. (2011). Parental involvement, child temperament, and parent's work hours: Differential relations for mothers and fathers. Journal of applied developmental psychology, 32(6), 313-322. http://dx.doi.org/10.1016/j. appdev.2011.08.004

Bueno, R. K., Gomes, L. B., \& Crepaldi, M. A. (2015). A importância do pai no desenvolvimento da criança. In E. R. Goetz e M. L. Vieira (Eds). Novo Pai: percursos, desafios e possibilidade (pp. 95-107).: Juruá.
Burney, R.V.,\&Leerkes, E.M.(2010).Linksbetweenmother's and father's perceptions of infant temperament and coparenting. Infant Behavior Development, 33(2), 125135. https://doi.org/10.1016/j.infbeh.2009.12.002

Caires, T. L. G., \& Vargens, O. M. C. (2012). A exclusão do pai da sala de parto: uma discussão de género e poder. Revista de Enfermagem Referência, III Série( $\mathrm{n}^{\circ}$ 7), 159-168. https://doi.org/10.12707/RIII1163

Casalin, S., Luyten, P., Vliegen, N., \& Meurs, P. (2012). The structure and stability of temperament from infancy to toddlerhood: A one-year prospective study. Infant Behavior and Development, 35(1), 94-108. https://doi. org/10.1016/j.infbeh.2011.08.004

Cassiano, R. G. M. (2013). A avaliação do temperamento em crianças: metodologia combinada de heterorrelato e observação do comportamento em situação de interação. (Dissertação de Mestrado, Universidade de São Paulo). Biblioteca Digital de Teses e Dissertações da USP. https://www.teses.usp.br/ teses/disponiveis/59/59137/tde-06052013-102556/ publico/Dissertacao_Rafaela_Guilherme_Monte_ Cassiano.pdf

Cerniglia, L., Cimino, S., \& Ballaroto, G. (2014). Motherchild and father-child interaction with their 24-monthold children during feeding, considering paternal involvement and the child's temperament in a community sample. Infant Mental Health Journal, 35(5), 473-481. https://doi.org/10.1002/imhj.21466

Cia, F., \& Barham, E. J. (2009). Envolvimento paterno e desenvolvimento social de crianças iniciando as atividades escolares. Psicologia em Estudo, 14(1), 67-74. https://doi.org/10.1590/S141373722009000100009

Consentino-Rocha, L., \& Linhares, M. B. M. (2013). Temperamento de Crianças e Diferença de Gênero. Paideia, 23(54), 63-72. https://doi.org/10.1590/198243272354201308

Cook, J. C., Schoppr-Sullivan, S. J., Buckley, C. K., \& Davis, E. F. (2009). Are some children harder to coparent than others? Children's negative emotionality and coparenting relationship quality. Journal of Family Psychology, 23(4), 606-610. https://doi.org/10.1037/ a0015992 
Dubeau, D., Devault, A., \& Paquette, D. (2009). L'engagement paternel, un concept aux multiples facettes. In D. Dubeau, A. Devault \& G. Forget (Eds.), La paternité au XXI sièle (pp 71-98). Les Presses de IUniversité Laval.

Else-Quest, N. M., Hyde, J. S., Goldsmith, H. H., \& Van Hulle, C. A. (2006). Gender differences in temperament: A meta-analysis. Psychological Bulletin, 132(1), 33-72. https://doi.org/10.1037/0033-2909.132.1.33

Flouri, E., \& Malmber, L-E. (2010). Child temperament and paternal transition to non-residence. Infant Behavior and Development, 33(4), 689-694. https://doi. org/10.1016/j.infbeh.2010.06.001

Goetz, E. R, \& Vieira, M. L. (2010). Pai real, pai ideal: o papel paterno no desenvolvimento infantil. Juruá.

Gracioli, S. M. A., \& Linhares, M. B. M. (2014). Temperamento e sua relação com problemas emocionais e de comportamento em pré-escolares. Psicologia em Estudo. 19(1). 71-80. https://doi. org/10.1590/1413-7372189590007

Guzzo, R. S. L., Riello, I. C., Primi, R., Serrano, M., Ito, P. C. P., \& Pinho, C. C. M. (2004). Temperamento: Onze anos de levantamento no psychological abstracts. Revista Estudos de Psicologia, 21(1), 25-32. https:// doi.org/10.1590/S0103-166X2004000100002

Hanington, L., Ramvhandani, P., \& Stein, A. (2010). Paternal depression and child temperament: Assessing child to parent effects in a longitudinal population study. Infant Behavior and Development, 33(1), 88-95. https:// doi.org/10.1016/j.infbeh.2009.11.004

Ito, P. C. P., \& Guzzo, R. S. L. (2002). Diferenças individuais: temperamento e personalidade; importância da teoria. Revista Estudos de Psicologia, 19(1), 91-100. https:// doi.org/10.1590/S0103-166X2002000100008

Karreman, A., Haas, S., Tuijl, C. V., Aken, M. A.G. V., \& Dekovic, M. (2010). Relations among temperament, parenting and problem behavior in young children. Infant Behavior and Development, 33(1), 39-49. https:// doi.org/10.1016/j.infbeh.2009.10.008
Kim, S., \& Kochanska, G. (2012). Child temperament moderates effects of parent-child mutuality on selfregulation: a relationship-based path for emotionally negative infants. Child Development, 83(4), 1275-1289. https://doi.org/10.1111/j.1467-8624.2012.01778.x

Kitamura, T., Ohashi, Y., Minatani, M., Haruna, M., Mutakami, M., \& Goto, Y. (2015). Disagreement between parents on assessment of child temperament traits. Pediatrics International, 57(6), 1090-1096. https://doi. org/10.1111/ped. 12728

Klein, V. C. (2009). Reatividade à dor, temperamento e comportamento na trajetória de desenvolvimento de neonatos pré-termo até a fase pré-escolar. (Tese de Doutorado, Universidade de São Paulo, Ribeirão Preto). Repositório da Produção USP. https://repositorio.usp. br/item/001791794

Klein, V. C., \& Linhares, M. B. M. (2007). Temperamento, comportamento e experiência dolorosa na trajetória de desenvolvimento da criança. Paidéia, 17(36), 33-44. https://doi.org/10.1590/S0103-863X2007000100004

Klein, V. C., e Linhares, M. B. M. (2010). Temperamento e desenvolvimento da criança: revisão sistemática da literatura. Psicologia em Estudo, 15(4), 821-829. https://doi.org/10.1590/s1413-73722010000400018

Kochanska, G., \& Kim, S. (2014). A complex interplay among the parent-child relationship, effortful control, and internalized, rule-compatible conduct in Young children: evidence from two studies. Developmental Psychopathology, 50(1), 8-21. https://doi.org/10.1037/ a0032330

Komsi, N., Raikkonen, K., Heinonen, K., Pesonen, A-K., Keskiaara, P., Jarvenpaa, A-L., \& Strandherg, T. E. (2008). Continuity of father temperament from infancy to middle childhood. ScienceDirect, 31(2), 239-254. https://doi.org/10.1016/j.infbeh.2007.10.002

Lamb, M. E. (1992). O papel do pai em mudança. Análise Psicológica, 1 (X), 19-34.

Lamb, M. E., Pleck, J. H., Charnov, E. L., \& Levine, J. A. (1985). Paternal Behavior in Humans. American Zoologist, 25(3), 883-894. https://doi.org/10.1093/ icb/25.3.883 
Lee, E. H., Zhou, Q., Eisenber, N., \& Wang, Y. (2013) Bidirectional relations between temperament and parenting styles in Chinese children. International Journal of Behavior Development, 37(1), 57-67. https:// doi.org/10.1177/0165025412460795

Linhares, M. B. M., Dualibe, A. L., \& Cassiano, R. G. M. (2013). Temperamento de crianças na abordagem de Rothbart: Estudo de revisão sistemática. Psicologia em Estudo, 18(4), 633-645. https://doi.org/10.1590/ s1413-73722013000400006

Malhado, S. C. B., \& Alvarenga, P. (2012). Relações entre temperamento infantil aos oito meses e as práticas educativas maternas aos 18 meses de vida da criança. Estudos de Psicologia, 29(1), 789-797. http:// dx.doi.org/10.1590/S0103-166X2012000500015

Martins, G. D. F., Becker, S. M. S., Leão, L. C. S., Lopes, R. C. S., \& Piccinini, C. A. (2014). Fatores associados à não adaptação do bebê na creche: da gestante ao ingresso na instituição. Psicologia: Teoria e Pesquisa, 30(3), 241-250. http://dx.doi.org/10.1590/S010237722014000300001

Matos, D. A. S. (2014). Confiabilidade e concordância entre juízes: aplicações na área educacional. Estudos em Avaliação Educacional. 25(59). 298-324. http:// dx.doi.org/10.18222/eae255920142750

Mehall, K. G., Spinrad, T. L., Eisenberg, N., \& Gaertner, B. M. (2009). Examining the relations of infant temperament and couples marital satisfaction to mother and father involvement: a longitudinal study. Fathering, 7(1), 2348. https://doi.org/10.3149/fth.0701.23

Miller, J. G., Chocol, C., Nuselovici, J. N., Utendale, W. T., Simard, M., \& Hastings, P. D. (2013). Children's dynamic RSA change during anger and its relations with parenting, temperament, and control of aggression. Biological Psychology, 92(2), 417-425. https://doi. org/10.1016/j.biopsycho.2012.12.005

Nelson, J. A. (2015). Child reactivity moderates the overtime association between mother-child conflict quality and externalizing problems. International Journal of Behavior Development, 39(4), 376-382. https://doi.org/10.1177/0165025415573643
Nogueira, J. R. D., \& Ferreira, M. (2012). O envolvimento do pai na gravidez/parto e a ligação emocional com o bebé. Revista de Enfermagem Referência, III Série(nº 8), 57-66. https://doi.org/10.12707/RIII1214

Oliveira, D. C. (2008). Análise de conteúdo temáticocategorial: Uma proposta de sistematização. Revista de Enfermagem, 16(4), 569-576.

Paquette, D. (2004a). Dichotomizing Paternal and Maternal Functions as a Means to Better Understand Their Primary Contributions. Human Development, 47, 237-238. https://doi.org/10.1159/000078726

Paquette, D. (2004). Theorizing the father-child relationship: mechanisms and developmental outcomes. Human Development, 47(4), 193-219. https://doi.org/10.1159/000078723

Paquette, D., \& Dumont, C. (2013). The Father-Child Activation Relationship, Sex Differences, and Attachment Disorganization in Toddlerhood. Child Development Research, 2013 1-9. https://doi. org/10.1155/2013/102860

Perdomini, F. R. I., \& Bonilha, A. L. L. (2011) A participação do pai como acompanhante da mulher no parto. Texto e Contexto Enfermagem, 20(3), 445-452. http://dx.doi. org/10.1590/S0104-07072011000300004

Planalp, E. M., \& Braungart-Rieker, J. M. (2013). Temperamental precursors of infant attachment with mothers and fathers. Infant Behavior and Development, 36(4), 796-808. https://doi.org/10.1016/j. infbeh.2013.09.004

Potapova, N. V., Gartstein, M. A., \& Bridgett, D. J. (2014). Paternal influences on infant temperament: Effects of father internalizing problems, parenting-related stress, and temperament. Infant Behavior and Development, 37(1), 105-110. https://doi.org/10.1016/j. infbeh.2013.12.014

Prior, M., Bavin, E. L., Cini, E., Reily, S., Bretherton, L., Wake, M., \& Eadie, P. (2008). Influences on communicative development at 24 months of age: Child temperament, behavior problems and maternal factors. Infant Behavior e Development, 31(2), 270-279. http://doi. org/10.1016/j.infbeh.2007.11.001 
Rabinowitz, J. A., Drabick, D. A. G., Reynolds, M. D., Clark, D. B., \& Olino, T. M. (2016). Child temperamental flexibility moderates the relation between positive parenting and adolescent adjustment. Journal of Applied Developmental Psychology, 43, 43-53. http:// doi.org/10.1016/j.appdev.2015.12.006

Rothbart, M. K. (2004). Commentary: Differentiated Measures of Temperament and Multiple Pathways to Child Disorders. Journal of Clinical Child and Adolescent Psychology, 33(1), 82-87. http://dx.doi. org/10.1207/S15374424JCCP3301_8

Rothbart, M. K., \& Hwang, J. (2002). Measuring infant temperament. Infant Behavior and Development, 25(1), 113-116. https://doi.org/10.1016/S01636383(02)00109-1

Sanson, A., Hemphill, S. A., \& Smart, D. (2004). Connections between temperament and social development: a review. Social Development, 13(1), 142-166. http://doi. org/10.1046/j.1467-9507.2004.00261.x

Seabra-Santos, M. J., \& Almeida, M. S. (2014). Falamos da mesma criança? Concordância mãe - pai professores na Avaliação do temperamento de crianças Portuguesas. Psicologia: Reflexão e Crítica, $27(1), \quad 10-20$. http://dx.doi.org/10.1590/S010279722014000100002

Silva, M. R. S., Bueno, M. E. N., \& Ribeiro, J. P. (2014). A percepção dos pais frente ao seu envolvimento nas atividades com o(s) filho(s). Revista Gaúcha de Enfermagem, 1, 14-21. http://dx.doi. org/10.1590/1983-1447.2014.01.36602

Silva, M. R., \& Piccinini, C. A. (2007). Sentimentos sobre a paternidade e o envolvimento paterno: um estudo qualitativo. Estudos de Psicologia, 24(4), 561-573. http://dx.doi.org/10.1590/S0103166X2007000400015
Schmidt, B. (2012). Relacionamento conjugal e temperamento de crianças com idade entre quatro e seis anos. (Dissertação de Mestrado, Universidade Federal de Santa Catarina). Repositório Institucional da UFSC. https://repositorio.ufsc.br/ handle/123456789/100663

Schmidt, B., Crepaldi, M. A., Vieira, M. L., \& Moré, C. L. O. O. (2011). Relacionamento conjugal e temperamento de crianças: uma revisão da literatura. Arquivos Brasileiros de Psicologia, 63(3), 89-106.

Schmidt, B., Gomes, L. B. G., Bossardi, C. N., Bolze, S. D. A., Vieira, M. L., \& Crepaldi, M. A. (2019). Envolvimento parental e temperamento de crianças: uma revisão sistemática da literatura. Contextos Clínicos, 12(1). https://doi.org/10.4013/ctc.2019.121.04

Turcotte, G., \& Gaudet, J. (2009). Conditions favorables et obstacles à l'engagement paternel: un bilan des connaissances. In D. Dubeau, A. Devault \& G. Forget (Eds.), La paternité au XXI siè (pp. 39-70.). Les presses de l'Université Laval.

Vieira, M. L., Bossardi, C. N., Gomes, L. B., Bolze, S. D. A., Crepaldi, M. A. \& Piccinini, C. A (2014). Paternidade no Brasil: revisão sistemática de artigos empíricos. Arquivos Brasileiros de Psicologia, 66(2). 36-52.

Data de submissão: 10/11/2017 Primeira decisão editorial: 21/03/2019 Aceite em 22/04/2019 